\title{
The role of aromatase inhibitors as adjuvant therapy in early breast cancer
}

\author{
A. U. Buzdar \\ Department of Medical Oncology, The University of Texas M.D. Anderson Cancer Center, Houston, TX, USA.
}

\begin{abstract}
Endocrine therapy is now established as the treatment option of choice for postmenopausal women with hormone receptor-positive advanced breast cancer. The benefit of the third-generation aromatase inhibitors (Als) in this setting has prompted their evaluation in the treatment of early breast cancer. This article reviews the current clinical status of Als as adjuvant therapy for early-stage breast cancer in postmenopausal women, focusing on the largest adjuvant breast cancer study till date to provide data, the 'Arimidex', Tamoxifen, Alone or in Combination (ATAC) trial.
\end{abstract}

Keywords: Aromatase inhibitors; Adjuvant therapy; Anastrozole; Early breast cancer; Exemestane; Letrozole; Postmenopausal women

\section{Introduction}

Approximately $75 \%$ of breast tumours in postmenopausal women are positive for the oestrogen receptor (ER) and/or progesterone receptor, and are potential candidates for endocrine treatment [1]. For more than 30 years, the non-steroidal-selective ER modulator tamoxifen has been the standard endocrine therapy for postmenopausal patients with breast cancer, although recently the long-term supremacy of tamoxifen has been challenged by the introduction of the third-generation aromatase inhibitors (Als). Indeed, Als have consistently demonstrated superiority over tamoxifen in the treatment of advanced breast cancer (reviewed in [2]).

Third-generation Als are potent inhibitors of the enzyme aromatase, which converts androgens to oestrogens in peripheral tissues (including malignant breast tissue), thereby suppressing production of oestrogen in postmenopausal women [3,4]. Currently

Correspondence to: Aman U. Buzdar, MD, Department of Medical Oncology, The University of Texas M.D. Anderson Cancer Center, 1515 Holcombe Blvd - 424, Houston, TX 77030 4009, USA. E-mail: abuzdar@mdanderson.org; Tel: +1 713792 2817; Fax: +1 7137944385

Publication date 27/10/04 BCO/49/2002/FO the value of Als in the early breast cancer setting is being evaluated in a number of clinical trials, via comparison directly with primary adjuvant tamoxifen, by switching to Al therapy after 2-3 years of prior adjuvant tamoxifen, or by comparing sequential therapy of 5 years of tamoxifen followed by an $\mathrm{Al}$ ('extended' adjuvant trials).

\section{Trials of Als as adjuvant therapy}

The 'Arimidex', Tamoxifen, Alone or in Combination trial

The 'Arimidex', Tamoxifen, Alone or in Combination (ATAC) trial is a large, international, randomized, double-blind, multicentre study that compares the efficacy of tamoxifen alone $(20 \mathrm{mg} ; n=3116)$ vs. anastrozole alone ( $1 \mathrm{mg} ; n=3125$ ) vs. a combination of both agents $(n=3125)$, as adjuvant treatment for postmenopausal women with early, operable breast cancer $[5,6]$.

\section{Efficacy}

First analysis

The first analysis (median duration follow-up of 33 months) showed a significant increase in disease-free 
survival (DFS; time to earliest occurrence of local or distant recurrence, new primary breast cancer or death from any cause) at 3 years with anastrozole (89.4\%) compared with tamoxifen $(87.4 \%)$ in the overall population, with a relative risk reduction of $17 \%$ (hazard ratio (HR) 0.83; 95\% confidence interval (Cl) 0.71, 0.96; $P=0.013$ ) [5]. Larger differences in relative risk reduction in DFS with anastrozole were observed in women with confirmed hormone receptor-positive cancer, who represented $84 \%$ of the total population and correspond to the target population for endocrine therapy (HR $0.78 ; 95 \% \mathrm{Cl}$ $0.65,0.93 ; P=0.005)$. For time to recurrence (TTR; including new contralateral tumours, but not including deaths from non-breast cancer causes before recurrence) anastrozole was also superior to tamoxifen in both the hormone receptor-positive subgroup (HR 0.73 ; $95 \% \mathrm{Cl} 0.59,0.90 ; P=0.003$ ) and the overall population (HR 0.79; 95\% Cl 0.67, 0.94; $P=0.008$ ). Overall, compared with tamoxifen, anastrozole was associated with a $58 \%$ reduction in the incidence of contralateral breast cancer (CLBC) (odds ratio 0.42; $95 \% \mathrm{Cl} 0.22,0.79 ; P=0.007$ ) [5]. First results from the ATAC trial did not show any significant differences between tamoxifen alone and the combination arm for any efficacy or safety endpoints analysed [5]; therefore, the combination arm was discontinued after this analysis, and is not considered here.

\section{Updated analysis}

An efficacy update conducted at a median follow-up of 47 months (when $46 \%$ of patients had been followed for at least 4 years), confirmed the superior efficacy of anastrozole over tamoxifen in terms of DFS and TTR, a difference that was more apparent in the clinically relevant hormone receptor-positive population (Table 1) [6]. The results of the updated efficacy analysis showed that the reduction in the incidence of CLBC also continued in the anastrozole group, and reached statistical significance in the subgroup of patients with hormone receptor-positive disease. Comparison of the absolute difference in DFS, and recurrence between the anastrozole and tamoxifen arms from the time of the first analysis to the updated analysis showed that the absolute benefit associated with anastrozole continued to increase with duration of follow-up (Table 2).

\section{Tolerability}

Data from the initial safety analysis (median treatment duration of 30.7 months) and from an updated safety analysis (median treatment duration of 36.9 months) showed that anastrozole was associated with a number of important tolerability benefits over tamoxifen $[5,6]$. Consistent with data from the initial
Table 1. Major efficacy endpoints at the updated analysis of the ATAC trial [6] (median follow-up of 47 months) for anastrozole compared with tamoxifen treatment.

\begin{tabular}{|c|c|c|}
\hline & HR (95\% Cl) & $P$-value \\
\hline \multicolumn{3}{|l|}{ DFS } \\
\hline Overall population & $0.86(0.76,0.99)$ & 0.03 \\
\hline $\begin{array}{l}\text { Hormone receptor- } \\
\text { positive population }\end{array}$ & $0.82(0.70,0.96)$ & 0.014 \\
\hline \multicolumn{3}{|l|}{ TTR } \\
\hline Overall population & $0.83(0.71,0.96)$ & 0.015 \\
\hline $\begin{array}{l}\text { Hormone receptor- } \\
\text { positive population }\end{array}$ & $0.78(0.65,0.93)$ & 0.007 \\
\hline \multicolumn{3}{|l|}{ Incidence of CLBC } \\
\hline Overall population & $0.62(0.38,1.02)^{a}$ & 0.06 \\
\hline $\begin{array}{l}\text { Hormone receptor- } \\
\text { positive population }\end{array}$ & $0.56(0.32,0.98)^{a}$ & 0.04 \\
\hline
\end{tabular}

Table 2. Absolute benefits in favour of anastrozole in terms of disease-free survival and disease recurrence after 3 and 4 years of treatment in the ATAC trial compared with tamoxifen $[5,6]$.

\begin{tabular}{lll}
\hline & \multicolumn{2}{l}{$\begin{array}{l}\text { Absolute difference between } \\
\text { anastrozole and tamoxifen (\%) }\end{array}$} \\
\cline { 2 - 3 } & 3 years & 4 years \\
\hline DFS & & \\
Overall population & 1.5 & 2.4 \\
Hormone receptor- & 1.7 & 2.9 \\
$\quad$ positive population & & \\
TTR & & \\
Overall population & 1.7 & 2.3 \\
Hormone receptor- & 1.7 & 2.6 \\
positive population & & \\
\hline
\end{tabular}

safety analysis, the updated analysis also demonstrated that more patients withdrew from treatment in the tamoxifen group compared with the anastrozole group ( $28.3 \%$ vs. $24.1 \%$, respectively) and fewer withdrawals were due to drug-related adverse events (AEs) ( $8.1 \%$ vs. $5.6 \%$, respectively). Tolerability benefits associated with anastrozole treatment included significant reductions in hot flushes, vaginal bleeding, vaginal discharge, endometrial malignancies, ischaemic cerebrovascular events, and venous thromboembolic events (including deepvein thromboses) (Fig. 1). Musculoskeletal disorders and fractures were significantly more common with anastrozole than with tamoxifen, although the relative risk of fractures or musculoskeletal disorders were unchanged between the initial and updated safety analyses [6]. Importantly, the fracture rate in the anastrozole-treated group stabilized after reaching a peak at 2 years [7]. It is possible that the effects of anastrozole on bone are magnified when compared 


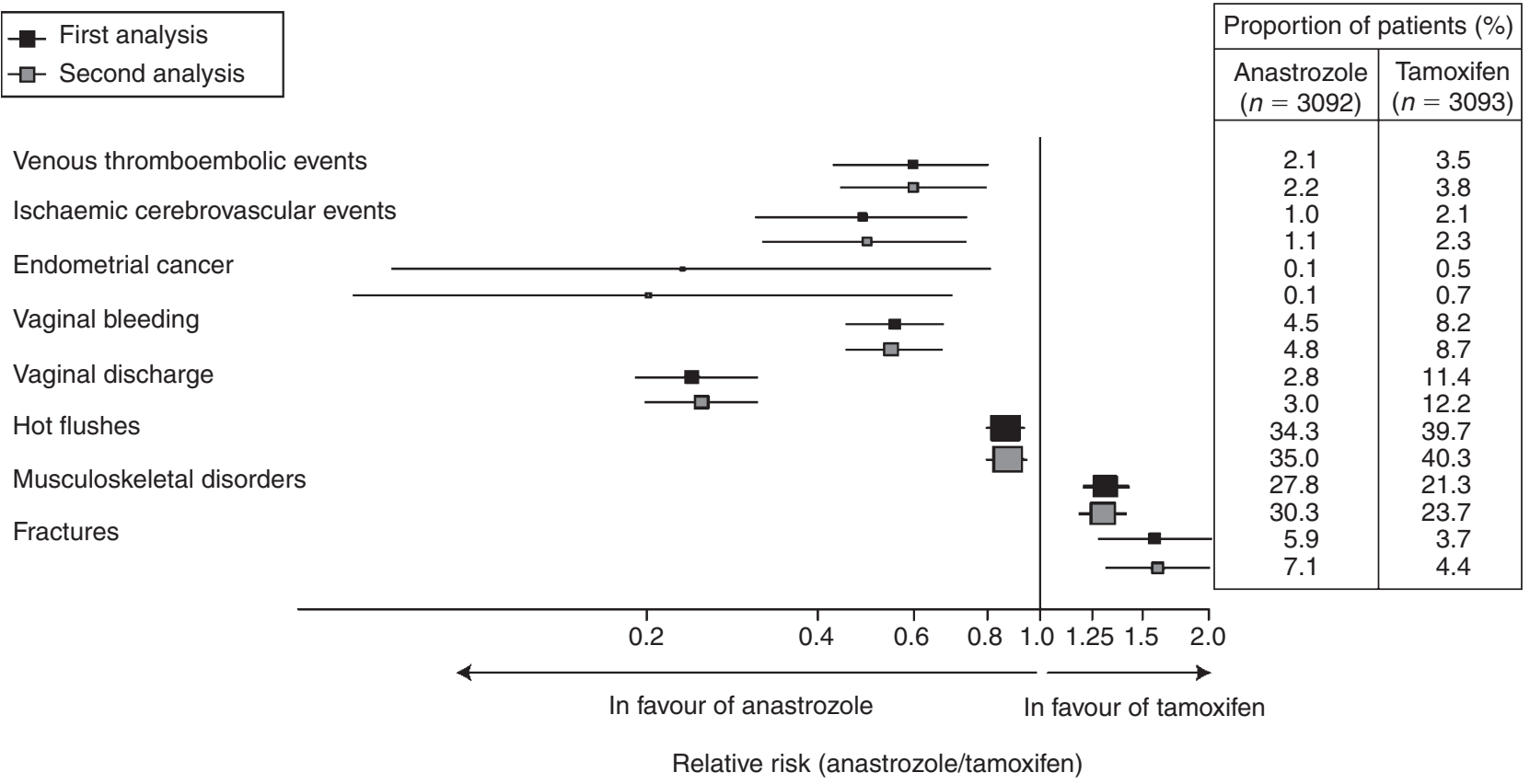

Figure 1.

Analysis of the predefined AEs (for which significant differences were observed between anastrozole and tamoxifen) in the first and updated analyses of the ATAC trial [5,6] showing the relative risk (95\% Cl) associated with anastrozole or tamoxifen treatment. The size of the boxes is proportional to the total number of observed AEs. The proportion of patients with AEs are also indicated.

with tamoxifen, which is known to increase bone mineral density (BMD) in postmenopausal women [8]. Bone loss can be monitored and patients at high risk of fracture should be managed according to local guidelines. Indeed, the impact of anastrozole on bone needs to be balanced against the overall efficacy and tolerability benefits observed in the main ATAC trial, and taken as a whole, the riskbenefit profile remains in favour of anastrozole.

\section{ATAC substudies}

Since the publication of the initial ATAC results, several substudies have provided further information on the potential long-term effects of oestrogen deprivation with anastrozole therapy with respect to endometrial abnormalities, bone and quality of life (QoL) [9-11]. An endometrial substudy conducted in 279 ATAC participants showed that tamoxifen was associated with a higher incidence of endometrial abnormalities than anastrozole alone [9]. The beneficial endometrial effects of anastrozole were confirmed in a separate analysis, which compared the incidence rates of endometrial cancer observed in the ATAC trial with those of an age-matched standard population, suggesting that anastrozole may have a protective effect on the endometrium [12].

A bone subprotocol of the ATAC trial has also been conducted. One year of anastrozole treatment was associated with a decrease in BMD in spine and hip, and an increase in bone resorption and bone formation markers, whereas the converse was observed with tamoxifen [10]. Updated BMD results from the ATAC study have shown the rate of bone loss with anastrozole was constant over 2 years of therapy [13].

In a QoL substudy of the ATAC trial, patients in all the three groups of the ATAC trial experienced some improvement in QoL over the 2 years of the analysis. QoL between the treatment groups was similar [11]. Differences in symptoms did suggest some advantage for anastrozole in terms of vaginal discharge and sweating compared with tamoxifen treatment, while tamoxifen was associated with fewer reports of painful intercourse and loss of sexual interest [14].

\section{Anastrozole or tamoxifen in the primary adjuvant setting?}

Results of the updated ATAC trial have shown for the first time that tamoxifen monotherapy is no longer the most effective endocrine treatment as primary adjuvant therapy in postmenopausal patients newly diagnosed with early breast cancer. The first 2 years of post-surgery represents the highest risk for breast cancer recurrence [15], making this period the most important for treatment [16]. Other clinical trials directly comparing either primary adjuvant letrozole 
(the Breast International Group (BIG) 01-98 study) or exemestane therapy (Tamoxifen Exemestane Adjuvant Multinational (TEAM) trial) with tamoxifen are currently ongoing and are expected to report their results in the near future.

\section{Other adjuvant aromatase inhibitor clinical trials}

\section{Anastrozole: the Intergruppo Tamoxifen Anastrozole (ITA) switching trial}

The ITA trial was a randomized, multicentre trial conducted to test the efficacy of switching postmenopausal patients who were already receiving adjuvant tamoxifen to anastrozole therapy. After 2-3 years of tamoxifen, patients were randomized to receive a further $2-3$ years of tamoxifen $(20 \mathrm{mg}$; $n=225)$ or switch to $2-3$ years of anastrozole (1 mg; $n=223)$, for a total duration of 5 years of endocrine therapy $[17,18]$. At a median follow-up of 36 months there was a significant difference in event-free survival $(P=0.0002)$ and recurrence-free survival $(P=0.001)$, which favoured the anastrozole treatment group. At the time of this analysis there was no significant difference in survival between treatment groups, probably due to the small number of deaths observed to date (10 and four in the tamoxifen and anastrozole groups, respectively).

The rate of discontinuation of treatment due to AEs was similar in the tamoxifen and anastrozole groups (4.4\% vs. $4.0 \%$, respectively) [18]. However, overall anastrozole was generally better tolerated than tamoxifen; switching to anastrozole resulted in a significant reduction in the incidence of gynaecological changes, including endometrial cancer $(0.9 \%$ vs. $7.1 \%$ for anastrozole and tamoxifen, respectively; $P=0.001$ ). For the anastrozole and tamoxifen groups, the incidence of joint/bone disorders $(7.2 \%$ vs. $5.8 \%$, respectively; $P=0.5$ ) and bone fractures $(0.9 \%$ vs. $0.9 \%$, respectively; $P=0.9)$ were not significantly different. Compared with tamoxifen, switching to anastrozole was associated with raised cholesterol levels $(2.7 \%$ vs. $8.1 \%$, respectively; $P=0.01)$ and increased gastrointestinal symptoms (1.3\% vs. $6.3 \%$, respectively; $P=0.006$ ). These differences may be attributed, at least in part, to the withdrawal of patients from tamoxifen, as other studies have shown anastrozole treatment to be lipid neutral [19]. However, further studies will be needed to clarify the effects of anastrozole on serum lipid profiles.

\section{Exemestane: BIG 97-02 switching trial}

The BIG 97-02 trial was a double-blind, randomized trial that was designed to evaluate switching adjuvant therapy to exemestane ( $25 \mathrm{mg} ; n=2362$ ) after
2-3 years of tamoxifen compared with continuing on tamoxifen (20 mg; $n=2380$ ) [20]. At a median follow-up of 30.6 months, exemestane significantly improved DFS compared with tamoxifen (HR 0.68; $95 \% \mathrm{Cl} 0.56,0.82 ; P<0.001$ ), representing a $32 \%$ reduction in risk and corresponding to an absolute benefit of $4.7 \%(95 \% \mathrm{Cl} 2.6,6.8)$ at 3 years after randomization. Results in ER-positive patients $(81 \%$ of the population) were very similar to those among all patients; exemestane demonstrated a $36 \%$ relative risk reduction in DFS. Exemestane also significantly reduced the risk of CLBC compared with tamoxifen, but was not associated with a survival benefit at this stage of the trial.

The analysis of AEs showed that, compared with tamoxifen, women who switched to exemestane experienced a significantly lower incidence of gynaecological symptoms, muscle cramps, and thromboembolic events (Table 3). Compared with tamoxifen, exemestane was associated with a significantly higher incidence of arthralgia and diarrhoea. In addition, switching to exemestane was associated with an increased incidence of osteoporosis and visual disturbances (Table 3), and a trend towards increased fractures $(2.3 \%$ vs. $3.1 \%$ for tamoxifen and exemestane, respectively; $P=0.08$ ). No significant differences were observed in the incidence of hot flushes, endometrial cancer, or cardiovascular disease (myocardial infarction was not included in this analysis) between treatment groups. Overall, more patients withdrew from treatment in the exemestane group compared with the tamoxifen group (365/2362 $(15.5 \%)$ vs. $302 / 2380$ (12.7\%), respectively).

Table 3. Incidence of adverse events during the BIG 97-02 trial for exemestane vs. tamoxifen [20] ${ }^{\text {a }}$. Reproduced with permission (Coombes et al. New Engl J Med 2004; 350: 1081-1092). Copyright (C) 2004 Massachusetts Medical Society. All rights reserved.

\begin{tabular}{|c|c|c|c|}
\hline & $\begin{array}{l}\text { Exemestane } \\
n(\%)\end{array}$ & $\begin{array}{l}\text { Tamoxifen } \\
n(\%)\end{array}$ & $P$-value \\
\hline Visual disturbances & $170(7.4)$ & $133(5.7)$ & 0.04 \\
\hline Osteoporosis & $171(7.4)$ & $134(5.7)$ & 0.05 \\
\hline $\begin{array}{l}\text { Gynaecological } \\
\text { symptoms }\end{array}$ & $135(5.8)$ & $211(9.0)$ & $<0.001$ \\
\hline Arthralgia & $124(5.4)$ & $85(3.6)$ & 0.01 \\
\hline Diarrhoea & $100(4.3)$ & $54(2.3)$ & $<0.001$ \\
\hline Vaginal bleeding & $93(4.0)$ & $129(5.5)$ & 0.05 \\
\hline Cramps & $64(2.8)$ & $102(4.4)$ & $<0.001$ \\
\hline $\begin{array}{l}\text { Thromboembolic } \\
\text { disease }\end{array}$ & $24(1.0)$ & $45(1.9)$ & 0.003 \\
\hline
\end{tabular}

a Incidence of adverse events for which significant differences $(P \leqslant 0.05)$ were observed between treatment groups is shown; ${ }^{\mathrm{b}} n=2305$ for exemestane; $n=2329$ for tamoxifen; data for gynaecological symptoms, osteoporosis and arthralgia were available for 2309 patients in the exemestane group and 2332 patients in the tamoxifen group. 
Although the overall survival and safety data from the BIG 97-02 and ITA trials $[17,20]$ have not yet reached maturity, data obtained to date suggest that 5 years of tamoxifen monotherapy no longer represents an optimal treatment choice. For women already part-way through their 5-year course of adjuvant tamoxifen, switching to anastrozole or exemestane therapy may therefore be more suitable. These studies only provide support for the concept of switching a patient to an Al part-way through a course of adjuvant tamoxifen and do not provide guidance as to which is the best treatment directly following surgery. At this point of time, it is inappropriate to speculate that switching from tamoxifen to an $\mathrm{Al}$ is superior to 5 years of anastrozole treatment alone, as these switching studies were not designed to directly compare these regimens. However, the ATAC study provides support for initiating adjuvant treatment with anastrozole, and the BIG 01-98 and the TEAM trials will provide data for letrozole and exemestane as initial adjuvant therapies in the future.

\section{Letrozole: The National Cancer Institute of Canada MA-17 'extended adjuvant' trial}

The MA-17 trial was a double-blind trial, designed to test the effectiveness of 5 years of letrozole therapy (2.5 mg; $n=2596$ ) vs. no further adjuvant treatment (placebo; $n=2594$ ) in postmenopausal women with ER-positive breast cancer who had completed 5 years of adjuvant tamoxifen [21]. Results from the first planned interim analysis from the MA-17 trial (median follow-up of 28.8 months) demonstrated that sequential treatment with 5 years of tamoxifen followed by letrozole reduced the risk of local or distant recurrence or new CLBC by $43 \%$ compared with 5 years of tamoxifen alone (letrozole: 75 new breast cancer events; placebo: 132 such events; HR $0.57 ; 95 \% \mathrm{Cl} 0.43,0.75 ; P \leqslant 0.001)$. Projected 4 -year DFS rates were $93 \%$ and $87 \%$ in the letrozole and placebo groups, respectively, based on data derived from approximately 50 patients who had been followed for the analysis of efficacy for $\geqslant 48$ months.

The MA-17 trial was stopped early on the recommendation of the Independent Data Monitoring Committee because of the significant DFS results at the first planned interim analysis. At this point, $<1 \%$ of patients had completed up to 4 years of follow-up and preliminary survival data showed that there was no treatment difference; the HR for death from any cause in the letrozole group compared with the placebo group was $0.76(95 \% \mathrm{Cl} 0.48,1.21 ; P=0.25)$ [21]. In general, letrozole was well tolerated, with $4.5 \%$ of women discontinuing treatment due to AEs compared with $3.6 \%$ in the placebo group $(P=0.11)$. Vaginal bleeding was significantly less frequent in the letrozole group compared with the placebo group $(P=0.01)$. However, several AEs were significantly increased in the letrozole group compared with the placebo group including: hot flushes (48\% vs. $40 \%$, respectively); arthralgia (21.3\% vs. $16.6 \%)$; and myalgia (11.8\% vs. $9.5 \%)(P<0.05$ in all cases). There was also a trend towards an increased rate of newly reported osteoporosis for women in the letrozole group compared with placebo $(5.8 \%$ vs. $4.5 \%$, respectively; $P=0.07$ ).

The MA-17 data are the first to show that patients who have completed 5 years of tamoxifen therapy may benefit from extended adjuvant therapy. It is not possible to determine whether the improved outcome in terms of DFS in the MA-17 trial with letrozole is due to the longer treatment period with endocrine therapy, to the sequential use of tamoxifen and letrozole, or to a combination of both. Early curtailment of this trial precludes analysis of mature survival data in the future, as a follow-up of patients in the placebo arm of the study will not be continued. In addition, the absence of more than 2 years of safety data overall and of any long-term safety data regarding fractures, osteoporosis and cardiovascular events means that the long-term risk : benefit profile of letrozole will not be provided.

The preliminary nature of data from the MA-17 trial highlights the need for long-term efficacy and tolerability data and information on overall survival [22]. These data should be provided for anastrozole and exemestane via ongoing trials in the extended adjuvant setting including the Austrian Breast Cancer Study Group, Study 6a (3 years anastrozole vs. placebo following 5 years of tamoxifen or tamoxifen plus aminoglutethimide).

\section{Revisiting the standard of care}

The ATAC trial is the first prospective trial to document the benefit of a third-generation $\mathrm{Al}$ in the adjuvant setting, and the only trial till date to have reported efficacy and safety data for primary adjuvant therapy with tamoxifen vs. anastrozole in patients newly diagnosed with early breast cancer $[5,6]$. Since there are many differences between Als in terms of pharmacokinetics, effects on lipid profiles and bone absorption, which may influence their long-term safety profiles [23], efficacy and safety data derived from the ATAC trial should not be extrapolated to other Als, as ratified by the American Society of Clinical Oncology (ASCO) Technology Assessment Working Group [24]. This report recommended that tamoxifen should still be used as the standard adjuvant endocrine therapy and that anastrozole should be considered to treat postmenopausal women with hormone receptor-positive tumours who have an 
absolute or relative contraindication to the use of tamoxifen [24]. However, the considerable experience gained with anastrozole in the ATAC trial to date, combined with further follow-up, should lead to the updating of treatment guidelines and clinical practice. Indeed, recent guidelines from the National Comprehensive Cancer Network consider anastrozole as an option to tamoxifen in postmenopausal women with hormone receptor-positive early breast cancer [25].

Results from the ITA trial [17] are in agreement with the overall results seen in ATAC, illustrating the efficacy and tolerability benefits of anastrozole over tamoxifen. However, direct comparison of results from the ATAC and ITA trials are inappropriate because of differences in trial design and demography of patient populations. Data from the BIG 97-02 trial [20] also show that 5 years of tamoxifen therapy may be suboptimal for use as adjuvant therapy in postmenopausal women with hormone receptor-positive cancer.

\section{Conclusion}

The third-generation Als promise an important clinical advance for postmenopausal women with early breast cancer. They offer improved efficacy over tamoxifen in a variety of adjuvant settings and also offer the potential for breast cancer prevention. Anastrozole is the only $\mathrm{Al}$ to date that has been approved for the adjuvant treatment of early breast cancer and the ASCO Technology Assessment Workgroup has already recommended the use of anastrozole in postmenopausal women with hormone receptor-positive early breast cancer who have an absolute or relative contraindication to tamoxifen. In addition, recent guidelines from the National Comprehensive Cancer Network consider anastrozole as an option to tamoxifen for the treatment of early breast cancer.

Ongoing clinical trials should help to define the precise timing, duration, and sequencing of Al therapy, in addition to the long-term tolerability profiles and potential differences between the Als. As the risk of breast cancer recurrence is highest during the first 5 years of adjuvant treatment post-surgery, and tamoxifen has been shown to be a suboptimal therapy for early or advanced disease, the initial choice between Al or tamoxifen may prove to be the pivotal treatment decision.

\section{Acknowledgements}

Sources of support in the form of grants: Research grants from AstraZeneca, Pfizer, Genentech and Taiho Pharmaceuticals.

\section{References}

1. Anderson WF, Chatterjee N, Ershler WB, Brawley OW. Estrogen receptor breast cancer phenotypes in the Surveillance, Epidemiology, and End Results database. Breast Cancer Res Treat 2002; 76: 27-36.

2. Buzdar AU. Aromatase inhibitors in breast cancer. Breast Cancer Online 2001; 4(4).

3. Cora EM, Maihle NJ. Aromatization of breast cancer. Breast Cancer Online 2002; 2(2).

4. Simpson ER, Dowsett M. Aromatase and its inhibitors: significance for breast cancer therapy. Recent Prog Horm Res 2002; 57: 317-338.

5. The ATAC Trialists' Group. Anastrozole alone or in combination with tamoxifen versus tamoxifen alone for adjuvant treatment of postmenopausal women with early breast cancer: first results of the ATAC randomised trial. Lancet 2002; 359: 2131-2139.

6. The ATAC Trialists' Group. Anastrozole alone or in combination with tamoxifen versus tamoxifen alone for adjuvant treatment of postmenopausal women with early-stage breast cancer. Results of the ATAC (Arimidex, Tamoxifen, Alone or in Combination) trial efficacy and safety update analyses. Cancer 2003; 98: 1802-2110.

7. Locker GY, Eastell R. The time course of bone fractures observed in the ATAC ('Arimidex', Tamoxifen, Alone or in Combination) trial. Proc Am Soc Clin Oncol 2003; 22: 25 [Abstract 98].

8. Ramaswamy B, Shapiro CL. Osteopenia and osteoporosis in women with breast cancer. Semin Oncol 2003; 30: 763-775.

9. Duffy S, Jackson T. Fewer endometrial abnormalities with anastrozole than tamoxifen: endometrial subprotocol results from the ATAC ('Arimidex', Tamoxifen, Alone or in Combination) early breast cancer (EBC) trial in postmenopausal (PM) patients (pts) (on behalf of the ATAC Trialists' Group). Ann Oncol 2002; 13(Suppl 5): 39 [Abstract 139P].

10. Eastell R, Adams J. Results of the 'Arimidex' (anastrozole, A), Tamoxifen (T), Alone or in Combination (C) (ATAC) trial: effects on bone mineral density (BMD) and bone turnover (ATAC Trialists' Group). Ann Oncol 2002; 13(Suppl 5): 32 [Abstract 113].

11. Fallowfield L, Cella D. Assessing the quality of life (QOL) of postmenopausal (PM) women randomized into the ATAC ('Arimidex', Tamoxifen, Alone or in Combination) adjuvant breast cancer (BC) trial. Proc Am Soc Clin Oncol 2002; 21: 40a [Abstract 159].

12. Duffy SR, Greenwood M. The endometrial cancer data from the ATAC ('Arimidex', Tamoxifen, Alone or in Combination) trial indicates a protective effect of anastrozole (Arimidex) upon the endometrium. Breast Cancer Res Treat 2003; 82(Suppl 1): S29.

13. Howell A. Effect of anastrozole on bone mineral density: 2-year results of the 'Arimidex' (anastrozole), Tamoxifen, Alone or in Combination (ATAC) trial. Breast Cancer Res Treat 2003; 82(Suppl 1): S27 [Abstract 129].

14. Fallowfield $L$. The importance of quality of life and patient preference in making treatment decisions in breast cancer. 8th International Breast Conference, 17-19 September 2003, Nottingham, UK.

15. Saphner T, Tormey DC, Gray R. Annual hazard rates of recurrence for breast cancer after primary therapy. $J$ Clin Oncol 1996; 14: 2738-2746. 
16. Early Breast Cancer Trialists' Collaborative Group. Tamoxifen for early breast cancer: an overview of the randomised trials. Lancet 1998; 351: 1451-1467.

17. Boccardo F, Rubagotti A, Amoroso D, et al. Anastrozole appears to be superior to tamoxifen in women already receiving adjuvant tamoxifen treatment. Breast Cancer Res Treat 2003; 82(Suppl 1): S6 [Abstract 3].

18. Boccardo F. Anastrozole appears to be superior to tamoxifen in women already receiving adjuvant tamoxifen treatment. http://hcp.arimidex.net/sites/68/imagebank/ typearticleparam501614/Boccardo\%20San\%20Antonio\% 202003\%20 ppt. 2003. Accessed 4 June 2004.

19. Sawada S, Sato K. Effect of anastrozole and tamoxifen on serum lipid levels in Japanese postmenopausal women with early breast cancer. Breast Cancer Res Treat 2003; 82(Suppl 1): S31-S32.

20. Coombes RC, Hall E, Gibson LJ, et al. A randomized trial of exemestane after two to three years of tamoxifen therapy in postmenopausal women with primary breast cancer. New Engl J Med 2004; 350: 1081-1092.
21. Goss PE, Ingle JN, Martino S, et al. A randomized trial of letrozole in postmenopausal women after five years of tamoxifen therapy for early-stage breast cancer. New Engl J Med 2003; 349: 1793-1802.

22. Buzdar AU. Letrozole in breast cancer. New Engl J Med 2004; 350: 727-730.

23. Buzdar A, Robertson JFR, Eiermann W, Nabholtz JM. An overview of the pharmacology and pharmacokinetics of the newer generation aromatase inhibitors anastrozole, letrozole and exemestane. Cancer 2002; 95: 2006-2016.

24. Winer EP, Hudis $\mathrm{C}$, Burstein $\mathrm{H}$, et al. Amercian Society of Clinical Oncology Technology Group Update: use of aromatase inhibitors in the adjuvant setting. $J$ Clin Oncol 2003; 21: 2597-2599.

25. National Comprehensive Cancer Network. Breast cancer. Clinical Practice Guidelines in Oncology, Vol. 1. 2004. www.nccn.org/physician_gls/f_guidelines.html 2004. Accessed 19 May 2004. 